
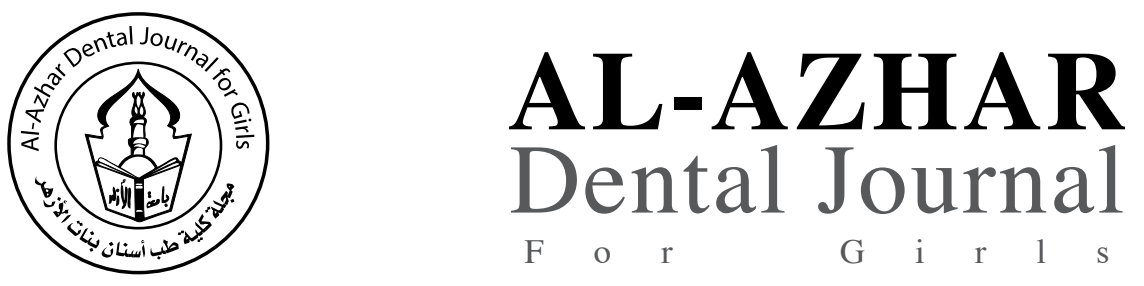

The Official Publication of The Faculty of Dental Medicine For Girls, Al-Azhar University Cairo, Egypt.

ADJ-for Grils, Vol. 4, No. 2, April (2017) — PP. 103:110

\title{
Evaluation of the Behavior Management Technique (Tell, Show and Do) and The Psychological Impact on the Child and Parents in Dental Practice
}

\section{Marwa El Karanshawy ${ }^{(1)}$, Samy Youssef El Bayoumy ${ }^{(2)}$ and Magdah El Malt ${ }^{(3)}$}

Codex : 12/1704

dentaljournal.forgirls@yahoo.com

Paper extracted from thesis titled Evaluation of the behavior management technique (Tell, show and do) and the psychological impact on the child and parents in dental practice.

\begin{abstract}
Objective: To evaluate the behavior management technique (Tell, show and do) and the psychological impact on the child and parents in dental practice. Material and Methods: In the present study 600 children were examined and assessed for the success of administration of local anesthesia in the dental office. The sample was divided into two main groups 300 in public practice and 300 in private practice. Within the 600 patients 400 patients were presented with the Tell, show and do technique directly by the dentist within the remaining 200 patients, 100 patients were presented the Tell, show and do technique by video presentation that was developed especially for this study. The remaining 100 patients were examined by the use of the voice control technique. The questionnaire that was done on all 600 patients was divided into pre-operative assessment, post-operative assessment, and Behavior rating scale. The pre-operative assessment included 17 different questions. The post-operative assessment included 14 different questions. The behavior rating scale included Frankl rating scale and anxiety rating scale. Results: The tell, show and do technique as well as the video presentation method showed to be more successful in the management of children during dental practice and demonstrated a higher level of success of administration of anesthesia in both genders. Tell show and do technique also is used more in the private sector rather than the public sector. Conclusion: Tell, show and do technique is a successful tool of behavior management technique in dental practice.
\end{abstract}

\section{KEYWORDS}

Behavior management,

Tell, show and do,

Psychological impact

1. B.D.S Misr International University $2006 \mathrm{G}$

2. Professor and Head of the Department of Pedodontics and Oral Public Health, Faculty of Dental Medicine(Boy's Branch), Al Azhar University.

3. Lecturer of Pedodontics and Oral Dental Health, Faculty of Dental Medicine (Girl's Branch) Al Azhar University. 


\section{INTRODUCTION}

Children and young adults, and indeed all patients, exhibit some form of anxiety or fear when about to receive dental care. However, effective behavior management can help alleviate the patient's fear or anxiety, an integral part of any dental practice that incorporates the care of children. Many factors have been proposed as contributory to children's anxiety about dental procedures. These include the dental clinic environment, equipment, past dental experience as well as attitude of the dental staff ${ }^{(1)}$

In order to achieve the co-operative child during dental treatment. It is necessary to attempt to modify or influence the child's behavior pattern. Therefore various behavior management strategies are being practiced to address these causative factors. These include verbal and nonverbal communication. Tell show and do, distraction, presence or absence of parents in the clinic, modeling, audio visual aids, positive reinforcements, physical restraints, hand over mouth exercise, sedation and general anesthesia. Behavior management methods in pediatric dentistry still either directed to maintain the communication process or intended to extinguish inappropriate behavior ${ }^{(2)}$

The selection of these behavior management techniques is no longer made solely by the dentist. In the past, dentists omitted parents from decisions regarding management of their child's behavior. Control has shifted from the health professional alone to more active involvement of the parent as well as several studies of parental acceptance of behavior management techniques used in pediatric dentistry offer differing views of parental awareness and attitudes ${ }^{(3)}$

It was reported that the dentist must realize the importance of home environment and parental influence upon the thinking and the behavior of the child. The parent not the child frequently becomes the major problem in implementing the treatment in the dental office. Children needs from parents love and affection, but at the same time they need firm guidance, they have to know what is permissible and what is not. Parent's attitude towards their child varies widely; it may be normal or slightly deviated from normal or even sometimes may be extremely abnormal. Parents with positive dental attitude will develop the same in the child. Whereas a fearful parent may develop fear unknowingly in a child ${ }^{(4)}$.

It was stated that fear plays an exceedingly important role in dentistry, especially in pediatric dentistry. Fear is the one of the primary emotions acquired soon after birth. However, the infant is unaware of the stimulus producing fear, as the child grows and his mental capacity increases he becomes aware of the stimuli producing fear and can identify each individually. Most of the time parents instill the fear of dentistry in their children as means of punishment. Fear should be channeled in the correct direction such as those that causes harm to the child's existence or wellbeing. Children should be taught that dental office isn't a place to fear, and the parents should never employ dentistry as threat ${ }^{(5)}$.

Communication is the key to behavior guidance and must be accomplished if the child is going to feel physically and emotionally secure, maintain self-esteem and feel responsible for behavior. Hints to aid in establishing communication with the child such as ignore initial demonstrations of uncooperativeness, begin with simple procedures that can be accomplished regardless of the kind of behavior exhibited, and then progress to more difficult ones. Moreover, avoid fear promoting by allowing the child to view sharp instruments, smell harsh chemicals, or hear the sounds of turbines until introduced to them properly, using fear-triggering vocabulary, shout, hurt, blood, cut, drill, needle, pull, over preparing the child and allowing too many questions, thus stalling the procedure; this only allows more anxiety build up, use Vocabulary that the child understands. The following are few examples of word substitutes that can be used when communicating with young children ${ }^{(6)}$. 
Table (1) Substitute terms for dental instruments (AAPD-2011)

\begin{tabular}{|l|l|}
\hline Dental Term & Substitute Term \\
\hline Explorer & Tooth counter \\
\hline Prophylaxis Paste & Tooth paste \\
\hline X- Ray equipment & Tooth Camera \\
\hline Impression material & Pudding \\
\hline Suction & Vacuum cleaner or straw \\
\hline Anesthesia & Sleepy juice for the tooth \\
\hline High speed turbine & Whistle \\
\hline Matrix band & Tooth ring \\
\hline Rubber Dam & Tooth rain coat \\
\hline Rubber Dam clamp & Tooth ring or button \\
\hline Stainless steel crown & Tooth hat \\
\hline Diagnostic model & Statues of Teeth \\
\hline
\end{tabular}

In order to understand dental anxiety and behavior management there are certain terminologist that will be used throughout this thesis section such as Dental fear (DF) - represents a reaction to a specific external threatening stimulus; this is a normal emotional reaction to threatening stimuli in the dental situation. Dental anxiety (DA) - represent a state where the child is evoked and prepared for something to happen; it is not attached to an object, instead a non-specific feeling of apprehension; this is associated with more abnormal conditions. Dental phobia - represents a severe type of dental anxiety; is characterized by marked and persistent fear of clearly discernible situations/objects; results in avoidance of necessary dental treatment or enduring treatment only with dread; interferes significantly with daily life. Dental behavior management problems (DBMP) - uncooperative and disruptive behaviors resulting in delay of treatment or rendering treatment impossible ${ }^{(7)}$.

Tell-show-do is a technique of behavior shaping used by many pediatric professionals. The technique involves verbal explanations of procedures in phrases appropriate to the developmental level of the patient (tell); demonstrations for the patient of the visual, auditory, olfactory, and tactile aspects of the procedure in a carefully defined, nonthreatening setting (show); and then, without deviating from the explanation and demonstration, completion of the procedure (do). The tell-show-do technique is used with communication skills (verbal and nonverbal) and positive reinforcement. The objectives of tellshow-do are to teach the patient important aspects of the dental visit and familiarize the patient with the dental setting; shape the patient's response to procedures through desensitization and well-described expectations ${ }^{(8)}$.

Voice control is a controlled alteration of voice volume, tone, or pace to influence and direct the patient's behavior. Parents unfamiliar with this technique may benefit from an explanation prior to its use to prevent misunderstanding. The objectives of voice control are to gain the patient's attention and compliance; avert negative or avoidance behavior; establish appropriate adult-child roles ${ }^{(9)}$.

Pediatric researchers are exhibiting a growing interest in evaluating techniques for managing the young child's negative behavior and reducing adverse emotional responses to treatment. One essential component of pediatric research program addressing these concerns is a reliable and well-validated tool for assessing the child's response to dental treatment. Unfortunately, few approaches have yet emerged to objectively observe and quantify the child's response in the dental setting. Therefore, the development of valid and reliable child assessment techniques is a major prerequisite for refining pediatric behavioral research and ultimately for improving the clinical management of children's dental anxiety and behavior. Behavioral rating scales have been the most commonly used indices of children's responses to dentistry ${ }^{(10)}$

An example is the widely used Frankl Scale, in which the child's reaction to dental treatment is rated on a four-point scale ranging from definitely negative to definitely positive. The advantage of 
rating scales include ease of administration and conceptualization. The rater uses the trait as an organizing concept which allows him or her to select relevant cues and to superimpose a dimension on the subject's behavior. Thus, the overall impression afforded by a rating may bring out a quality or unity to the child's behavior that a tabulation of discrete behaviors may be unable to reveal. In assigning ratings, the rater is able to take account of individual response styles in behavior and to consider infrequent but significant behaviors ${ }^{(11)}$.

The major drawbacks of the rating procedure lie in possible undetected bias and distortion of data. The scorer weighs the evidence on which the rating is based on a complex manner which is not easily specified, standardized, or objectified. Subjective factors, such as the ego-involvement or expectations of the rater may lead to scoring bias. To the degree that the rating process remains unstandardized and subjective, reliability and validity may be compromised. The possibility of rater bias, and the associated reliability and validity problems, are maximized when scaling categories are not defined in clear cut behavioral terms. In the commonly used Frankl Scale, an effort was made to define scale points behaviorally. Nonetheless, the definitions remain sufficiently subjective that latitude exists for each rater to interpret the scale categories somewhat idiosyncratically ${ }^{(12)}$

\section{METHODS AND TECHNIQUES}

When the child arrives for the first appointment, a thorough history and examination should be completed. This will assist in a definitive diagnosis and allow for logical preventive and treatment objectives to be developed with the parent and the child. A systematic approach to examination, diagnosis, and treatment planning will be described and although it is easy to follow such an outline, it isn't advised. Such an approach does not lend itself to the meaningful interpersonal relationships between patient and doctor that are necessary in a successful private practice. Therefore the following outline serves only as a guide for the type of information that should be obtained ${ }^{(13)}$.

In light of the coloration and the importance of the clinical examination and the bond created between the child and the dentist, as well as the fact that the data collected in this questionnaire is helpful to assess the history of the child especially if he/ she had a previous painful experience in the dental clinic. The questionnaire formulated for this study relayed on three main sections a preoperative analysis or the parent questionnaire and then the operative analysis and the anxiety and behavior measure as well as the Frankl scale for the local anesthesia ${ }^{(14)}$.

This study was designed to measure the level of success of administration of anesthesia with minimum stress and anxiety. The questionnaire was conducted in both public and private sector so as to measure the difference of the socio-economic factor. The questionnaire was done by myself as well as the presence of the assistance so as to ensure the elimination of any bias in the results. The Tell, show and do or the voice was conducted after the preoperative questionnaire and then the post-operative as well as the scales was conducted after the administration of the anesthesia the scores was done by both dentists in reelection to child reaction and ease of administration of anesthesia. The entire questionnaire was reviewed again to ensure the proper calculation of the results.

The entire procedure was repeated with each patient to ensure the unifying of the results. The same procedure was handled in the video presentation so as to demonstrate the tell, show and do technique. The entire procedure was explained to the parent as well as the child to ensure the understanding of the procedure as well as the aim of the questionnaire.

\section{RESULTS}

In the present study 600 children were examined and assessed for the success of administration of local anesthesia in the dental office. The sample was divided into two main groups 300 in public 
practice and 300 in private practice. Within the 600 patients 400 patients were presented with the Tell, show and do technique directly by the dentist within the remaining 200 patient, 100 patients were presented the Tell, show and do technique by video presentation that was developed especially for this thesis. The remaining 100 patients were examined by the use of the voice control technique. The questionnaire that was done on all 600 patients was divided into pre-operative assessment, post-operative assessment, and Behavior rating scale. The pre-operative assessment included 17 different questions. The post-operative assessment included 14 different questions. The behavior rating scale included Frankl rating scale and anxiety rating scale.

This study was done from September 2013 till January 2015. Six hundred Egyptian children were randomly selected from two different hospitals the Azhar University (Girl's Branch) hospital and also Cairo University dental hospital, paid section. The age range between 6 years and 12 years old; the patients were girls and boys of the same age divided between three techniques, Tell show and do and voice control and also video presentation of the tell show and do technique. The questionnaire was fabricated for three sections pre- operative assessment of the patients, post-operative assessment and a behavior rating scale and frankle rating scale to measure the success of the administration of the anesthesia. The statistical comparison was conducted between the females and males patient of the same age group. Also it was taken into consideration the patient's presence and the previous dental experiences as well the past dental visit and the nature of the visit.

Data were expressed as number and percentages and was used for generation of tables and figures. Both groups were compared using Chi Square test. The significance level was set at $p \leq 0.05$. Statistical analysis was performed with SPSS 16.0 (Statistical Package for Scientific Studies, SPSS, Inc., Chicago, IL, USA) for Windows.

\section{DISCUSSION}

It was noted through the course of this study that children who experience bad dental treatment weather males and females demonstrated uncooperative behavior in there next appointment and were refusing treatment this was demonstrated with $89 \%$ of patients in this study with painful dental experience where considered uncooperative. These finding correlate with the Guide line on Behavioral guidance of which states that children with previous unpleasant dental or medical experience may significantly affect a child's subsequent ability to accept dentistry, while children with positive medical experience may be less apprehensive to treatment. The study contained a postoperative assessment discussing the success of administrating the anesthesia as well as the child behavior and level of cooperation with the dentist as well as the parent impression towards the technique used to modify the child behavior ${ }^{(15)}$.

In regards to the child behavior in the dental visit among the male patients $57 \%$ were cooperative among female patients $66 \%$ were cooperative which indicated great significance difference, this also seem in coloration with the response to verbal commands question which showed that females tend to comply more to verbal commands. It was noticed that in males boys 8 years and above are more cooperative while in females starting 7 years old tend to be cooperative at younger age different to males. There was no significance difference between the public and private sector when it came to child behavior it matched the results of the gender and age groups.

In regards to the treatment plan module which was the Tell, show and do technique which was demonstrated by the dentist to the patients and explained as well to the parents accompanying the child $91 \%$ of the males where satisfied with the treatment and also $79 \%$ of the females were satisfied with the treatment plan. The same situation 
applies for the private and public practice were total of $89 \%$ of the patients were satisfied with the child management technique offered to their children.

In regards to requesting another treatment plan for their children $91 \%$ of the males were satisfied with their treatment plan and same $79 \%$ of the females were also satisfied with the treatment plan the same applied for the private and public sector they both demonstrated $92 \%$ satisfaction with the treatment plan. Those results also relates to the technique offering them a pleasant experience in there dental visit almost $97 \%$ of the male patient stated they had a pleasant experience and $93 \%$ of the female patients stated they had a pleasant experience also the same applies for the public as well as the private sector $94 \%$ of the patients reported to have a pleasant experience as well as their parents. This result agrees with the finding, which stated that Tell, show and do is a technique using several concepts from a learning theory that it is well accepted technique by parents and it works well combined with behavioral shaping, it is a technique that can be used with any age group and showed zero contraindication. It was mentioned in several research that explaining the treatment to the parents accompanying the children helped to reduce child anxiety in dental treatment ${ }^{(16)}$.

In regards to the time consuming factor, among both males and females patient the procedure was perceived as not time consuming and also the same applied on the private and public sector the percentage of $92 \%$ of the patient perceived the procedure as a not time consuming. This factor is a critical one that when dealing with children both the time and length of the appointment are important. Children cannot sit in one position for longer time and their threshold of tolerance is very low. Therefore, they should not be kept in the chair for periods longer than half an hour. With longer appointment they tend to become less cooperative. Once a child loses his self-composure his cooperative is very difficult to regain ${ }^{(17)}$.
In the public sector $79 \%$ of the patients and their parents stated that the tell, show and do technique wasn't explained to them before as well as in the private sector almost $97 \%$ of the patients were familiar with the tell, show and do technique. Those results stated that the compliance of parents in the use of management technique is affected by their socioeconomic status and that $93 \%$ of doctors relay on verbal commands and are not adopting new communication technique to manage children in dental practice. In regards to the overall judgment of the treatment in males and females it was find that in the anxiety scale $40 \%$ of the males were at level 1 and $39 \%$ were at level 0 and the females $47 \%$ were at level 1 and 24\% were at level 0 these scores were set according the appearance and reaction of the child the moment he enters the dental clinic to meet his dentist $^{(18)}$

In regards to the acceptance of administration of anesthesia in both females and males it was successful with $94 \%$ to be able to administer the anesthesia at Rate 4 which states that the child is defiantly positive and good rapport with the dentist and interested in the dental procedure and laughing and enjoying the situation. Those scores come in coloration with the use of behavioral management technique which showed the success of the tell, show and do in managing the child patient in dental practice ${ }^{(19)}$

In this section will be discussing the results of the remaining 200 patients, 100 patients were presented the Tell, show and do technique by video presentation that was developed especially for this thesis. The remaining 100 patients were examined by the use of the voice control technique. In regards to their reaction in preoperative assessment. The statistical analysis was done in cross comparison between both techniques as well as in the private and public sectors.

In regards to child behavior in dental visit, $87 \%$ of the children presented with the video presentation were cooperative and only $2 \%$ were uncooperative in comparison to $45 \%$ of patients given the voice 
control were uncooperative and only $11 \%$ were cooperative this reveled a highly significant difference in the chi square analysis between the two groups.

In regards to the treatment plan, in $77 \%$ of children receiving the video technique the experience was pleasant, while in $23 \%$ it was not pleasant. On the other hand, only $5 \%$ of children receiving the voice technique were pleased with the experience. Chi-square test revealed that the difference between the 2 groups was highly significant $(\mathrm{P}<0.0001)$.In the same domain the regards to the pleasance of the experience $77 \%$ of the patient receiving the video presentation stated that it was a pleasant experience and helped with reducing the anxiety while patient receiving the voice control stated by $65 \%$ that it wasn't a pleasant experience and that $71 \%$ of them preferred a different treatment plan, while patient receiving the video presentation stated by $95 \%$ that they don't want another treatment plan. The above findings come in coloration with the fact that voice control may seem to work with some patients it is considered unacceptable with most parents and it isn't useful with all children and tend to result in unpleasant experience to patients as well as care givers and it isn't appropriate for young children and children with emotional impairment ${ }^{(20)}$.

In regards to the behavioral rating scales there was a significant difference in all the scales used between the voice control and the video presentation of the tell, show and do technique. In regards to the anxiety scale, $80 \%$ of children receiving the video technique were scale $1,15 \%$ were scale 0 . On the other hand, $36 \%$ of children receiving the voice technique were scale 2, $25 \%$ were scale 5 and $16 \%$ were scale 3 .

In regards to the behaviour scale, $80 \%$ of children receiving the video technique were scale 1 , $14 \%$ were scale 0 . On the other hand, $40 \%$ of children receiving the voice technique were scale 2 , $20 \%$ were scale 5 and $19 \%$ were scale 1 . In regards to Frankl scale, $94 \%$ of children receiving the video technique were rate $4,6 \%$ were rate 2 . On the other hand, $39 \%$ of children receiving the voice technique were rate $1,30 \%$ were rate 3 and $26 \%$ were rate 2 .

The above finding stated that a short video presentation of treatment or explanation of procedure to be conducted have been shown to reduce the disruptive behavior compared to the control group that received a verbal command technique. It is was also reported to highly reduce fear in children experiencing dental treatment for the first time and also helped to reduce fear in patients undergoing treatment for the second time and also it seem to be highly effective in children aged 5 to 9 years old ${ }^{(21)}$.

The above finding also relates to study finding that the use of dentist tone of voice to control the patient seem to establish dental fear rather than ease it. It is the strengthening of a pattern of behavior by removal of a stimulus which the patient perceive as unpleasant rather than reinforcing it and replacing the verbal negative command with a positive one and praising the child for good behavior rather than highlighting negative one seem to ease dental anxiety and enhance the success of the dental treatment ${ }^{(22)}$.

\section{CONCLUSION}

- Effective behavior management technique can help alleviate the patient's fear and anxiety.

- The use of tell, show and do was highly acceptable by most patients as well as parents and was perceived to be a pleasant experience and also helped to reduce the dental anxiety in both the patient as well as their parents.

- The use of behavior management technique is essential to eliminate the causative factors of dental fear and anxiety. Children with pleasant dental experiences have been seen to be more cooperative and also accepting of dental treatment.

- The level of anxiety of parents or care givers accompanying the child have been seen to be highly significant and affect the child behavior during the dental visit and also it is seen to be passed to the child during the visit. 
- The use of the voice control technique was refused by most parents and also seen as time consuming and exhausting to both parent and child and was also in most cases unsuccessful in behavior modification.

- The video presentation used explaining the instruments used during the visit showed to be highly significant in reducing dental anxiety and aiding in the behavior modification of the child. The use of such technique was highly acceptable by parents and also children as a tool to communicate with them and explain the procedure.

\section{REFERENCES}

- 1-Abushal MS., ADbenubi,J.O: The use of behavior management techniques by dentists in Saudi Arabia: A survey: Saudi DJ 2012;12:129-34

- 2-Adair SM: Behavior management conference panel I report- Rationale for behavior management techniques in pediatric dentistry. Pediatric Dent 2004; 26:167-70.

- 3-Eaton JJ, McTigue DJ, Fields HW Jnr, Beck M. Attitudes of contemporary parents towards behavior management techniques used in pediatric dentistry. Pediatric Dent, 2005; 27:107-13.

- 4- Foster T, Perinpanayagam H, Pfaffenbach A, Certo M. Recurrence of early childhood caries after comprehensive treatment with general anesthesia and follow up. J Dent Child (Chic) 2006:73(1):25-30.

- 5-Howard KE, Freeman R. An evaluation of the PALS after treatment modeling interventions to reduce dental anxiety in child dental patients. Int J Paed Dent, 2009; 19: 233-42.

- 6-Assuncão CM, Losso EM, Andreatini R, de Menezes $\mathrm{JV}$. The relationship between dental anxiety in children, adolescents and their parents at dental environment. J Indian Soc Pedod Prev Dent 2013;31:175-9.

- 7-Abushal MS., ADbenubi,J.O: The use of behavior management techniques by dentists in Saudi Arabia: A survey: Saudi DJ 2012;12:129-34

- 8-American Academy of Pediatric Dentistry, clinical affairs committee: Policy on alternative restorative treatment (ART). Pediatr Dent 2006; 28(suppl):33

- $\quad$ 9-Kotsans N, Arhakis A, Coolideg T. Parental presences versus absence in the dental operatory; a technique to manage the uncooperative child dental patient. Eur J Paedatr Dent 2004; 14(1): 2-8.

- Onody P. Modification of behavior with 50\%Nitorus oxide/oxygen conscious sedation over repeated visits for dental treatment a 3 years prospective study. J Clin Psychopharmacology 2006;26(5):474-81.

- $\quad$ Adair SM,Schafer TE, Rockman RA, Waller JL. Survey of behavior management teaching in predoctoral pediatric dentistry program. Pediatr Dent 2004: 26(2):143-50.

- $\quad$ 12- Frankel R1. The Paposse Board and mother's attitude following its use. Pediatr Dent. 1991; 13: 284-8

- 13- Sheller B. Challenges of managing child behavior in the 21st century dental setting. Pediatr Dent 2004; 26(2):111-3

- 14-Adair SM, Rockman RA, Schafer TE, Waller JL. Survey of behavior management teaching in pediatric dentistry advanced education programs. Pediatr Dent 2004:26(2):151-8

- 15- American Academy of Pediatric Dentistry. Guideline on Behavior Guidance for the Pediatric Dental Patient. Pediatr Dent 2011; 36:161-73.

- 16-American Academy of Pediatric Dentistry: Guideline on pediatric restorative dentistry. Pediatric Dent, 2009; 28(suppl):136-43.

- 17-Adair SM, Rockman RA, Schafer TE, Waller JL. Survey of behavior management teaching in pediatric dentistry advanced education programs. Pediatr Dent 2004:26(2):151-8

- 18- Paryab M, Hosseinbor M. Dental anxiety and behavioral problems: a study of prevalence and related factors among a group of Iranian children aged 6-12. J Indian Soc Pedod Prev Dent 2013;31:82-6

- 19-Abushal MS., ADbenubi,J.O: The use of behavior management techniques by dentists in Saudi Arabia: A survey: Saudi DJ 2012;12:129-34

- 20-Abushal MS., Adenubi JO.: Attitudes of Saudi Parents towards behavior management techniques in pediatric dentistry. J Dent Child 2003; 70(2):104-10.

- 21-American Academy of Pediatric Dentistry, clinical affairs committee: Policy on alternative restorative treatment (ART). Pediatr Dent 2006; 28(suppl):33

- 22-Adair SM, Rockman RA, Schafer TE, Waller JL. Survey of behavior management teaching in pediatric dentistry advanced education programs. Pediatr Dent 2004:26(2):155-8 